\title{
Article \\ Convergence Results for the Double-Diffusion Perturbation Equations
}

\author{
Jincheng Shi ${ }^{1, *}$ and Shiguang Luo ${ }^{2}$ \\ 1 School of Data Science, Guangzhou Huashang College, Guangzhou 511300, China \\ 2 Department of Applied Mathematics, Guangdong University of Finance, Guangzhou 510521, China; \\ 26-047@gduf.edu.cn \\ * Correspondence: shijc0818@gdhsc.edu.cn
}

check for updates

Citation: Shi, J.; Luo, S. Convergence Results for the Double-Diffusion Perturbation Equations. Symmetry 2022, 14, 67. https://doi.org/ $10.3390 /$ sym 14010067

Academic Editor: Aviv Gibali

Received: 29 November 2021

Accepted: 17 December 2021

Published: 4 January 2022

Publisher's Note: MDPI stays neutral with regard to jurisdictional claims in published maps and institutional affiliations.

Copyright: (c) 2022 by the authors. Licensee MDPI, Basel, Switzerland. This article is an open access article distributed under the terms and conditions of the Creative Commons Attribution (CC BY) license (https:/ / creativecommons.org/licenses/by/ $4.0 /)$.

\begin{abstract}
We study the structural stability for the double-diffusion perturbation equations. Using the a priori bounds, the convergence results on the reaction boundary coefficients $k_{1}, k_{2}$ and the Lewis coefficient $L_{e}$ could be obtained with the aid of some Poincaré inequalities. The results showed that the structural stability is valid for the the double-diffusion perturbation equations with reaction boundary conditions. Our results can be seen as a version of symmetry in inequality for studying the structural stability.
\end{abstract}

Keywords: structural stability; double-diffusion perturbation equations; Lewis coefficient; convergence result

\section{Introduction}

Many papers in the literature have studied the continuous dependence or convergence of solutions of different equations in porous media on construction coefficients. We give these studies a new name. We call these stabilities structural stability. This kind of stability is different from the traditional stability. We do not care about the stability with the initial data, but about their structural stability with the model itself. For an introduction to the nature of this structural stability, please see book [1]. It is important to establish the result of the structural stability in the problem of the continuum mechanics. In [2], the authors studied a variety of equations and obtained many results on structural stability. We think it is very important to study structural stability. In the process of establishing the model, the error always exists. We want to know whether a small error will cause a sharp change in the solution

Straughan in paper [3] proposed a new type of double diffusion perturbation model in porous media. The Darcy approximation is used in the derivation of this type of equation. We usually call this type of equation Darcy equations. Details about such types of equations were introduced in $[4,5]$.

There are many equations that describe fluids in porous media. In books $[4,6,7]$, the authors studied many different types of equations. In [8-10], the saint-venant principle results were studied for the Brinkman, Darcy, and Forchheimer equations. The spatial decay results were obtained. In the literature, many results on the structural stability of equations in porous media have been obtained. Representative papers can be seen by [11-16]. It should be emphasized that some new results have also emerged recently, see [17-48]. These results all belong to the category of the study of structural stability.

In this article, we continue to consider the structural stability of such types of equations. We consider the following double-diffusion perturbation equations with velocity, pressure, temperature, and concentration perturbations: 


$$
\left\{\begin{array}{l}
\frac{\partial u_{i}}{\partial t}=C \varphi l_{i}-R \theta l_{i}+\pi_{, i}, \\
\frac{\partial u_{i}}{\partial x_{i}}=0, \\
\frac{\partial \theta}{\partial t}+u_{i} \theta_{, i}=u_{3}+\Delta \theta, \\
\varepsilon_{1} \frac{\partial \varphi}{\partial t}+L_{e} u_{i} \varphi_{, i}=u_{3}+\Delta \varphi,
\end{array}\right.
$$

where $u_{i}, \theta, \varphi$, and $\pi$ are the velocity, temperature, concentration disturbance, and pressure, respectively. $\Delta$ is the Laplace operator. In Equation (1), $R$ is the Rayleigh coefficient and $C$ is the salinity Rayleigh coefficient, $\varepsilon_{1}$ represents the porosity, and $L_{e}$ is the Lewis coefficient, $l=(0,0,1)=\left(l_{1}, l_{2}, l_{3}\right)$. The system of Equation (1) is established in the region $\Omega \times[0, \tau]$, where $\Omega$ is bounded in the strictly convex region in $R^{3}$, and $\tau$ is a given constant and satisfies $0 \leq \tau<\infty$. The boundary conditions are:

$$
u_{i} n_{i}=0, \quad \frac{\partial \theta}{\partial n}=k_{1} \theta, \quad \frac{\partial \varphi}{\partial n}=k_{2} \varphi, \quad(x, t) \in \partial \Omega \times[0, \tau] .
$$

The initial conditions are:

$$
u_{i}(x, 0)=u_{i 0}(x), \varphi(x, 0)=\varphi_{0}(x), \theta(x, 0)=\theta_{0}(x), x \in \Omega .
$$

There are significant differences between the double-diffusion perturbation equations and the Brinkman, Forchheimer, Darcy equations. The main difficulty is that we can not get the maximum value of the disturbance as the previous papers [11-16]. In the references, the maximum value of the disturbance is often used to obtain the required structural stability results. In this paper, we can not get the maximum estimates of disturbance. The structural stability results we need will not be obtained by using the previous methods. We must adopt a new method to overcome the difficulty of not getting the maximum value. We adapt the $L^{4}$ norm of the disturbance. In addition, since the velocity equation does not contain the Laplacian term, the estimates of the gradient of the velocity is not easy to obtain. There is no Laplacian term, so we can not get the gradient estimation of the velocity according to the conventional method. The estimation of gradient of the velocity is very important in this paper. How to get the gradient estimation of the velocity is the biggest innovation of this paper because the space and the inequalities used in this paper have the property of symmetry. Our results can be regarded as the application of the symmetry in the study of the structural stability. In this article, we will use other estimations to obtain the gradient estimation. The content of this paper is arranged as follows: First, some a prior estimates of the solutions are given, and then based on these a prior estimates, the differential inequality satisfied by the concentration difference of the solution is established, and the convergence results of the solutions are obtained by integrating the inequality. The following notational conventions are adopted in the text: A comma is used to indicate the partial derivative. For example, $i$ denotes the partial differentiation with respect to $x_{i}$. For example $u_{, i}=\frac{\partial u}{\partial x_{i}}$. The repeated Latin subscripts denotes summation. For example, $u_{i, i}=\sum_{i=1}^{3} \frac{\partial u_{i}}{\partial x_{i}}$. The symbol $d x=d x_{1} d x_{2} d x_{3}$.

\section{A Priori Bounds}

In the course of producing the results of convergence on the coefficient of (1), we find it is easy if we can derive some a priori bounds for the solutions. We will give some Lemmas that are useful in proving our main results.

Lemma 1. For the temperature $\theta$ and the concentration disturbance $\varphi$, we have the following estimates:

$$
\int_{\partial \Omega} \theta^{2} d S \leq\left(\frac{m_{1}}{m_{0}}+\frac{\varepsilon_{0} m_{2}^{2}}{m_{0}^{2}}\right) \int_{\Omega} \theta^{2} d S+\frac{1}{\varepsilon_{0}} \int_{\Omega} \theta_{, i} \theta_{, i} d S,
$$


and

$$
\int_{\partial \Omega} \varphi^{2} d S \leq\left(\frac{m_{1}}{m_{0}}+\frac{\varepsilon_{0} m_{2}^{2}}{m_{0}^{2}}\right) \int_{\Omega} \varphi^{2} d S+\frac{1}{\varepsilon_{0}} \int_{\Omega} \varphi_{, i} \varphi_{, i} d S,
$$

where $m_{0}, m_{1}, m_{2}$ are positive constants, and $\varepsilon_{0}$ is an arbitrary positive constant.

Proof. We defined a function $\xi_{i}$ on $\Omega$. The function $\xi_{i}$ satisfies the following conditions:

$$
\begin{gathered}
\xi_{i} n_{i} \geq m_{0}>0, x \in \partial \Omega, \\
\left|\xi_{i, i}\right| \leq m_{1}, \mathrm{x} \in \Omega, \\
\left|\xi_{i}\right| \leq m_{2}, \mathrm{x} \in \Omega,
\end{gathered}
$$

where $n_{i}$ is the unit outward normal vector.

From the divergence theorem, we have:

$$
\begin{aligned}
m_{0} \oint_{\partial \Omega} \theta^{2} d s & \leq \oint_{\partial \Omega} \xi_{i} n_{i} \theta^{2} d s \\
& =\int_{\Omega}\left(\xi_{i} \theta^{2}\right)_{i} d x \\
& =\int_{\Omega} \xi_{i, i} \theta^{2} d x+2 \int_{\Omega} \xi_{i} \theta \theta_{, i} d x \\
& \leq m_{1} \int_{\Omega} \theta^{2} d x+2 m_{2} \int_{\Omega} \theta \bar{x} \theta d x .
\end{aligned}
$$

Using Schwarz's inequality, we have:

$$
\oint_{\partial \Omega} \theta^{2} d s \leq\left(\frac{m_{1}}{m_{0}}+\frac{\varepsilon_{0} m_{2}^{2}}{m_{0}^{2}}\right) \int_{\Omega} \theta^{2} d x+\frac{1}{\varepsilon_{0}} \int_{\Omega} \theta_{, i} \theta, i d x .
$$

Following the same procedures, we can also get:

$$
\int_{\partial \Omega} \varphi^{2} d S \leq\left(\frac{m_{1}}{m_{0}}+\frac{\varepsilon_{0} m_{2}^{2}}{m_{0}^{2}}\right) \int_{\Omega} \varphi^{2} d S+\frac{1}{\varepsilon_{0}} \int_{\Omega} \varphi_{, i} \varphi_{, i} d S .
$$

Lemma 2. For the velocity $u_{i}$, temperature $\theta$, and the concentration disturbance $\varphi$, we have the following estimates:

$$
\begin{gathered}
\int_{\Omega} u_{i} u_{i} d x+\int_{\Omega} \theta^{2} d x+\int_{\Omega} \varphi^{2} d x \leq n_{1}(t), \\
\int_{0}^{t} \int_{\Omega} \theta_{, i} \theta_{, i} d x d \eta \leq n_{2}(t), \\
\int_{0}^{t} \int_{\Omega} \varphi_{, i} \varphi_{, i} d x d \eta \leq n_{3}(t),
\end{gathered}
$$

where $n_{1}(t), n_{2}(t)$, and $n_{3}(t)$ are non-negative monotonically increasing functions.

Proof. Multiplying both sides of the Equation (1) by $2 u_{i}$, and integrating over $\Omega$, we can get:

$$
\begin{aligned}
\frac{d}{d t} \int_{\Omega} u_{i} u_{i} d x & =2 C \int_{\Omega} \varphi l_{i} u_{i} d x-2 R \int_{\Omega} \theta l_{i} u_{i} d x+2 \int_{\Omega} \pi_{, i} u_{i} d x \\
& \leq 2 \int_{\Omega} u_{i} u_{i} d x+C^{2} \int_{\Omega} \varphi^{2} d x+R^{2} \int_{\Omega} \theta^{2} d x .
\end{aligned}
$$


Multiplying both sides of the Equation (1) by $2 \theta$, and integrating over $\Omega$, we can get:

$$
\frac{d}{d t} \int_{\Omega} \theta^{2} d x=2 \int_{\Omega} u_{3} \theta d x+2 \int_{\Omega} \theta \Delta \theta d x-2 \int_{\Omega} u_{i} \theta_{i} \theta d x .
$$

Using (4) and taking $\varepsilon_{0}=2 k_{1}$, we can get:

$$
\int_{\partial \Omega} \theta^{2} d S \leq\left[\frac{m_{1}}{m_{0}}+\frac{2 k_{1} m_{2}^{2}}{m_{0}^{2}}\right] \int_{\Omega} \theta^{2} d x+\frac{1}{2 k_{1}} \int_{\Omega} \theta_{, i} \theta_{, i} d x .
$$

For the second term on the right side of Equation (16), we have:

$$
\begin{aligned}
2 \int_{\Omega} \theta \Delta \theta d x & =2 \int_{\partial \Omega} \theta \frac{\partial \theta}{\partial n} d S-2 \int_{\Omega} \theta_{, i} \theta_{i} d x \\
& =2 k_{1} \int_{\partial \Omega} \theta^{2} d S-2 \int_{\Omega} \theta_{, i} \theta_{, i} d x \\
& \leq\left(\frac{2 k_{1} m_{1}}{m_{0}}+\frac{4 k_{1}^{2} m_{2}^{2}}{m_{0}^{2}}\right) \int_{\Omega} \theta^{2} d x-\int_{\Omega} \theta_{, i} \theta_{, i} d x
\end{aligned}
$$

Combining (16) and (18), and using the Hölder's inequality, we can get:

$$
\frac{d}{d t} \int_{\Omega} \theta^{2} d x+\int_{\Omega} \theta_{, i} \theta_{, i} d x \leq \int_{\Omega} u_{i} u_{i} d x+\left(\frac{2 k_{1} m_{1}}{m_{0}}+\frac{4 k_{1}^{2} m_{2}^{2}}{m_{0}^{2}}+1\right) \int_{\Omega} \theta^{2} d x .
$$

Multiplying both sides of Equation (1) by $2 \varphi$, and integrating over $\Omega$, we can obtain:

$$
\begin{aligned}
& \varepsilon_{1} \frac{d}{d t} \int_{\Omega} \varphi^{2} d x+\int_{\Omega} \varphi_{, i} \varphi_{, i} d x \\
& \leq \int_{\Omega} u_{i} u_{i} d x+\left(\frac{2 k_{2} m_{1}}{m_{0}}+\frac{4 k_{2}^{2} m_{2}^{2}}{m_{0}^{2}}+1\right) \int_{\Omega} \varphi^{2} d x .
\end{aligned}
$$

We define a new function:

$$
F_{1}(t)=\int_{\Omega} u_{i} u_{i} d x+\int_{\Omega} \theta^{2} d x+\int_{\Omega} \varphi^{2} d x
$$

Combining (15), (19), and (20), we obtain:

$$
F_{1}(t) \leq m_{4}+m_{3} \int_{0}^{t} F_{1}(\eta) d \eta
$$

where

$$
m_{3}=\max \left\{3+\frac{1}{\varepsilon_{1}}, R^{2}+\frac{2 k_{1} m_{1}}{m_{0}}+\frac{4 k_{1}^{2} m_{2}^{2}}{m_{0}^{2}}+1, C^{2}+\frac{2 k_{2} m_{1}}{m_{0} \varepsilon_{1}}+\frac{4 k_{2}^{2} m_{2}^{2}}{m_{0}^{2} \varepsilon_{1}}+\frac{1}{\varepsilon_{1}}\right\},
$$

and

$$
m_{4}=\int_{\Omega} u_{i 0} u_{i 0} d x+\int_{\Omega} \theta_{0} \theta_{0} d x+\int_{\Omega} \varphi_{0} \varphi_{0} d x
$$

Using Gronwall's inequality, we can get:

$$
F_{1}(t) \leq m_{3} m_{4} e^{m_{3} t} \int_{0}^{t} e^{-m_{3} \eta} d \eta=n_{1}(t) .
$$

Inserting (22) into (19), we can get:

$$
\int_{0}^{t} \int_{\Omega} \theta_{, i} \theta_{i} d x d \eta \leq\left(\frac{2 k_{1} m_{1}}{m_{0}}+\frac{4 k_{1}^{2} m_{2}^{2}}{m_{0}^{2}}+1\right) \int_{0}^{t} n_{1}(\eta) d \eta=n_{2}(t) .
$$


Inserting (22) into (20), we can get:

$$
\int_{0}^{t} \int_{\Omega} \varphi_{, i} \varphi_{, i} d x d \eta \leq\left(\frac{2 k_{2} m_{1}}{m_{0}}+\frac{4 k_{2}^{2} m_{2}^{2}}{m_{0}^{2}}+1\right) \int_{0}^{t} n_{1}(\eta) d \eta=n_{3}(t) .
$$

Lemma 3. For velocity $u_{i}$, we have the following estimates:

$$
\left[\int_{\Omega}\left(u_{i} u_{i}\right)^{2} d x\right]^{\frac{1}{2}} \leq m_{1}(t)
$$

where $m_{1}(t)$ is a positive function to be defined later.

Proof. We have the following identity:

$$
\int_{\Omega} u_{i, j} u_{i, j} d x=\int_{\Omega} u_{i, j}\left(u_{i, j}-u_{j, i}\right) d x+\int_{\Omega} u_{i, j} u_{j, i} d x
$$

Since $\partial \Omega$ is bounded, we know from the result of [44]:

$$
\left|\int_{\Omega} u_{i, j} u_{j, i} d x\right| \leq k_{0} \int_{\partial \Omega} u_{i} u_{i} d s,
$$

where $k_{0}$ is the Gaussian curvature depending on $\partial \Omega$.

Taking $\theta=u_{i}, \varepsilon_{0}=2 k_{0}$ in (4), we have:

$$
\begin{aligned}
\left|\int_{\Omega} u_{i, j} u_{j, i} d x\right| & \leq k_{0}\left(\frac{m_{1}}{m_{0}}+\frac{2 k_{0} m_{2}^{2}}{m_{0}^{2}}\right) \int_{\Omega} u_{i} u_{i} d x+\frac{1}{2} \int_{\Omega} u_{i, j} u_{i, j} d x \\
& \leq k_{0}\left(\frac{m_{1}}{m_{0}}+\frac{2 k_{0} m_{2}^{2}}{m_{0}^{2}}\right) n_{1}(t)+\frac{1}{2} \int_{\Omega} u_{i, j} u_{i, j} d x
\end{aligned}
$$

Combining (24) and (25), we get:

$$
\int_{\Omega} u_{i, j} u_{j, i} d x \leq 2 \int_{\Omega} u_{i, j}\left(u_{i, j}-u_{j, i}\right) d x+2 k_{0}\left(\frac{3}{m}+\frac{2 k_{0} d^{2}}{2 m^{2}}\right) n_{1}(t) .
$$

Using Equation (1), we obtain:

$$
\begin{aligned}
& \frac{d}{d t} \int_{\Omega} u_{i, j}\left(u_{i, j}-u_{j, i}\right) d x=2 \int_{\Omega}\left(u_{i, j}-u_{j, i}\right) u_{i, j t} d x \\
& \leq 4 \int_{\Omega} u_{i, j}\left(u_{i, j}-u_{j, i}\right) d x+C^{2} \int_{\Omega} \varphi_{, j} \varphi_{, j} d x+R^{2} \int_{\Omega} \theta_{, j} \theta_{, j} d x .
\end{aligned}
$$

We define $E(t)=\int_{\Omega} u_{i, j}\left(u_{i, j}-u_{j, i}\right) d x$. From (26), we obtain:

$$
E(t) \leq 4 e^{4 t} \int_{0}^{t} m(y) e^{-4 y} d y+R^{2} n_{2}(t)+C^{2} n_{3}(t)=m_{2}(t),
$$

with $m(t)=\int_{\Omega} u_{i, j}(x, 0)\left[\left(u_{i, j}(x, 0)-u_{j, i}(x, 0)\right] d x\right.$.

Inserting (28) into (26), we have:

$$
\int_{\Omega} u_{i, j} u_{i, j} d x \leq 2 m_{2}(t)+2 k_{0}\left(\frac{m_{1}}{m_{0}}+\frac{2 k_{0} m_{2}^{2}}{m_{0}^{2}}\right) n_{1}(t) .
$$


Using the result of (B.17) in [26], we have:

$$
\begin{aligned}
& \left(\int_{\Omega}|u|^{4} d x\right)^{\frac{1}{2}} \leq M\left(\frac{5}{4} \int_{\Omega}|u|^{2} d x+\frac{3}{4} \int_{\Omega}|\nabla u|^{2} d x\right) \\
& \leq M\left(\frac{5}{4} n_{1}(t)+\frac{6}{4} m_{2}(t)\right)+\frac{6 k_{0}}{4}\left(\frac{m_{1}}{m_{0}}+\frac{2 k_{0} m_{2}^{2}}{m_{0}^{2}}\right) n_{1}(t)=m_{1}(t)
\end{aligned}
$$

where $M$ is a positive constant.

Lemma 4. For the temperature $\theta$, concentration disturbance $\varphi$, we have the following estimates:

$$
\begin{aligned}
& \left(\int_{\Omega} \theta^{4} d x\right)^{\frac{1}{2}} \leq n_{4}(t), \\
& \left(\int_{\Omega} \varphi^{4} d x\right)^{\frac{1}{2}} \leq n_{5}(t),
\end{aligned}
$$

with $n_{4}(t)$ and $n_{5}(t)$ are all monotonically increasing functions greater than zero.

Proof. Multiplying both sides of the $(1)_{3}$ by $\theta^{3}$ and integrating over $\Omega$, we have:

$$
\begin{aligned}
\frac{1}{4} \frac{d}{d t} \int_{\Omega} \theta^{4} d x+\int_{\Omega} u_{i} \theta_{, i} \theta^{3} d x & =\int_{\Omega} u_{3} \theta^{3} d x+\int_{\Omega} \theta^{3} \theta_{, i i} d x \\
& =\int_{\Omega} u_{3} \theta^{3} d x-3 \int_{\Omega} \theta^{2} \theta_{, i} \theta_{, i} d x+k_{1} \int_{\partial \Omega} \theta^{4} d S \\
& \leq \frac{1}{4} \int_{\Omega} u_{3}^{4} d x+\frac{3}{4} \int_{\Omega} \theta^{4} d x-3 \int_{\Omega} \theta^{2} \theta_{, i} \theta_{, i} d x+k_{1} \int_{\partial \Omega} \theta^{4} d x
\end{aligned}
$$

Replacing $\theta$ by $\theta^{2}$ and choosing $\varepsilon_{0}=2 k_{1}$ in (4), we get:

$$
k_{1} \int_{\partial \Omega} \theta^{4} d x \leq \frac{k_{1} m_{1}}{m_{0}} \int_{n} \theta^{4} d x+\frac{2 k_{1}^{2} m_{2}^{2}}{m_{0}^{2}} \int_{n} \theta^{4} d x+\frac{1}{2} \int_{\Omega}\left(\theta^{2}\right)_{, i}\left(\theta^{2}\right)_{, i} d x .
$$

Inserting (34) into (33), we obtain:

$$
\frac{d}{d t} \int_{\Omega} \theta^{4} d x \leq \int_{\Omega} u_{3}^{4} d x+3 \int_{n} \theta^{4} d x+\frac{4 m_{1} k_{1}}{m_{0}} \int_{n} \theta^{4} d x+\frac{8 k_{1}^{2} m_{2}^{2}}{m_{0}^{2}} \int_{n} \theta^{4} d x .
$$

Inserting (23) into (35), we obtain:

$$
\frac{d}{d t} \int_{\Omega} \theta^{4} d x \leq m_{1}^{2}(t)+\left(3+\frac{4 m_{1} k_{1}}{m_{0}}+\frac{8 k_{1}^{2} m_{2}^{2}}{m_{0}^{2}}\right) \int_{n} \theta^{4} d x .
$$

An integration of (36) leads to

$$
\int_{\Omega} \theta^{4} d x \leq e^{\left(3+\frac{4 m_{1} k_{1}}{m_{0}}+\frac{8 k_{1}^{2} m_{2}^{2}}{m^{2}}\right) t}\left[\int_{\Omega} \theta_{0}^{4} d x+\int_{0}^{t}\left(m_{1}(\eta)\right)^{2} d \eta\right] .
$$

We obtain:

$$
\left(\int_{n} \theta^{4} d x\right)^{\frac{1}{2}} \leq n_{4}(t)
$$

Following the same procedures, we can also get:

$$
\left(\int_{\Omega} \psi^{4} d x\right)^{\frac{1}{2}} \leq n_{5}(t)
$$


with

$$
n_{4}(t)=e^{\left(3+\frac{4 m_{1} k_{1}}{m_{0}}+\frac{8 k_{1}^{2} m_{2}^{2}}{m_{0}^{2}}\right) t}\left[\int_{\Omega} \theta_{0}^{2} d x+\int_{0}^{t}\left(m_{1}(\eta)\right)^{2} d \eta\right],
$$

and

$$
n_{5}(t)=e^{\left(3+\frac{4 m k_{2}}{m_{0}}+\frac{8 k_{2}^{2} m_{2}^{2}}{m_{0}^{2}}\right) t}\left[\int_{\Omega} \psi^{4} d x+\int_{0}^{t}\left(m_{1}(\eta)\right)^{2} d \eta\right] .
$$

\section{Convergence Result for the Reaction Boundary Coefficients $k_{1}$ and $k_{2}$}

Let $\left(u_{i}, \theta, \varphi, \pi\right)$ be the solution of (1)-(3) with $k_{1}=\hat{k_{1}}, k_{2}=\hat{k_{2}}\left(u_{i}^{*}, \theta^{*}, \varphi^{*}, \pi^{*}\right)$ be the solution of (1)-(3) with $k_{1}=0, k_{2}=0$. We define $\omega_{i}=u_{i}-u_{i}^{*}, \hat{\theta}=\theta-\theta^{*}, \hat{\varphi}=\varphi-\varphi^{*}$, $\hat{\pi}=\pi-\pi^{*}$, then $\left(\omega_{i}, \hat{\theta}, \hat{\varphi}, \hat{\pi}\right)$ satisfies the following equations:

$$
\begin{array}{r}
\frac{\partial \omega_{i}}{\partial t}=C \hat{\varphi} l_{i}-R \hat{\theta} l_{i}+\hat{\pi}_{, i} \\
\frac{\partial \omega_{i}}{\partial x_{i}}=0 \\
\frac{\partial \hat{\theta}}{\partial t}+\omega_{i} \theta_{, i}+u_{i}^{*} \hat{\theta}_{, i}=\omega_{3}+\Delta \hat{\theta}, \\
\varepsilon_{1} \frac{\partial \hat{\varphi}}{\partial t}+L_{e}\left(\omega_{i} \varphi_{, i}+u_{i}^{*} \hat{\varphi}_{, i}\right)=\omega_{3}+\Delta \hat{\varphi} .
\end{array}
$$

The boundary conditions are:

$$
\omega_{i} n_{i}=0, \frac{\partial \hat{\theta}}{\partial n}=\hat{k_{1}} \theta, \frac{\partial \hat{\varphi}}{\partial n}=\hat{k_{2}} \varphi,(x, t) \in \partial \Omega \times[0, \tau] .
$$

The initial conditions are:

$$
\omega_{i}(x, 0)=0, \hat{\varphi}(x, 0)=0, \hat{\theta}(x, 0)=0, x \in \Omega .
$$

In deducing our main result, we will use the following Lemma.

Lemma 5. For the difference of the velocity $\omega_{i}$, we can get the following estimates:

$$
\int_{\Omega} \omega_{i, j} \omega_{i, j} d x \leq 2 \int_{\Omega} \omega_{i, j}\left(\omega_{i, j}-\omega_{j, i}\right) d x+2 k_{0}\left[\frac{m_{1}}{m_{0}}+\frac{2 k_{0} m_{2}^{2}}{m_{0}^{2}}\right] \int_{\Omega} \omega_{i} \omega_{i} d x,
$$

with $k_{0}$ as a positive constant.

Proof. We know the fact:

$$
\int_{\Omega} \omega_{i, j} \omega_{i, j} d x=\int_{\Omega} \omega_{i, j}\left(\omega_{i, j}-\omega_{j, i}\right) d x+\int_{\Omega} \omega_{i, j} \omega_{j, i} d x .
$$

Since the boundary of $\Omega$ is bounded, we have:

$$
\left|\int_{\Omega} \omega_{i, j} \omega_{j, i} d x\right| \leq k_{0} \int_{\partial \Omega} \omega_{i} \omega_{i} d S,
$$

with $k_{0}$ as a positive constant depending on the Gaussian curvature of $\partial \Omega$ (see [44]).

Using the result (4) with $\varepsilon_{0}=2 k_{0}$, we can obtain:

$$
\int_{\partial \Omega} \omega_{i} \omega_{i} d S \leq\left[\frac{m_{1}}{m_{0}}+\frac{2 k_{0} m_{2}^{2}}{m_{0}^{2}}\right] \int_{\Omega} \omega_{i} \omega_{i} d x+\frac{1}{2 k_{0}} \int_{\Omega} \omega_{i, j} \omega_{i, j} d x .
$$


Inserting (45) and (46) into (44), we can get:

$$
\int_{\Omega} \omega_{i, j} \omega_{i, j} d x \leq 2 \int_{\Omega} \omega_{i, j}\left(\omega_{i, j}-\omega_{j, i}\right) d x+2 k_{0}\left[\frac{m_{1}}{m_{0}}+\frac{2 k_{0} m_{2}^{2}}{m_{0}^{2}}\right] \int_{\Omega} \omega_{i} \omega_{i} d x .
$$

In this part, we will get the following Theorem.

Theorem 1. Let $\left(u_{i}, \theta, \varphi, \pi\right)$ be the classical solution of the initial value problem (1)-(3) with $k_{1}=\hat{k_{1}}, k_{2}=\hat{k_{2}}$, and $\left(u_{i}^{*}, \theta^{*}, \varphi^{*}, \pi^{*}\right)$ be the classical solution of the initial boundary value problem (1)-(3) with $k_{1}=0, k_{2}=0 . \quad\left(\omega_{i}, \hat{\theta}, \hat{\varphi}, \hat{\pi}\right)$ is the difference of these two solutions. When $\hat{k_{1}}$ and $\hat{k_{2}}$ tend to zero, the solution $\left(u_{i}, \theta, \varphi, \pi\right)$ converges to the solution $\left(u_{i}^{*}, \theta^{*}, \varphi^{*}, \pi^{*}\right)$. The difference of the solution $\left(\omega_{i}, \hat{\theta}, \hat{\varphi}, \hat{\pi}\right)$ satisfies:

$$
\begin{aligned}
& \int_{\Omega} \omega_{i} \omega_{i} d x+\int_{\Omega} \hat{\theta}^{2} d x+\varepsilon_{1} \int_{\Omega} \hat{\varphi}^{2} d x+\int_{\Omega} \omega_{i, j}\left(\omega_{i, j}-\omega_{j, i}\right) d x \\
& \leq \hat{k}_{1}^{2} m_{8} e^{m_{8} t} n_{6}(t)+\hat{k}_{2}^{2} m_{8} e^{m_{8} t} n_{7}(t),
\end{aligned}
$$

where $m_{8}$ is a positive constant and $n_{6}(t)$ and $n_{7}(t)$ are positive functions.

Proof. Multiplying both sides of Equation (40) by $2 \omega_{i}$, and integrating over $\Omega$, we can get:

$$
\begin{aligned}
\frac{d}{d t} \int_{\Omega} \omega_{i} \omega_{i} d x & =2 C \int_{\Omega} \hat{\varphi} l_{i} \omega_{i} d x-2 R \int_{\Omega} \hat{\theta} l_{i} \omega_{i} d x+2 \int_{\Omega} \hat{\pi}_{i} \omega_{i} d x \\
& \leq 2 \int_{\Omega} \omega_{i} \omega_{i} d x+C^{2} \int_{\Omega} \hat{\varphi}^{2} d x+R^{2} \int_{\Omega} \hat{\theta}^{2} d x
\end{aligned}
$$

From Equation (40), we know:

$$
\begin{aligned}
& \frac{d}{d t} \int_{\Omega} \omega_{i, j}\left(\omega_{i, j}-\omega_{j, i}\right) d x \\
& =2 \int_{\Omega}\left(\omega_{i, j}-\omega_{j, i}\right) \omega_{i, j t} d x \\
& =2 C \int_{\Omega}\left(\omega_{i, j}-\omega_{j, i}\right) \hat{\varphi}_{, j} l_{i} d x+2 \int_{\Omega}\left(\omega_{i, j}-\omega_{j, i}\right) \hat{\pi}_{, i j} d x-2 R \int_{\Omega}\left(\omega_{i, j}-\omega_{j, i}\right) \hat{\theta}_{, j} l_{i} d x .
\end{aligned}
$$

Using the divergence theorem and Hölder's inequality, we can get:

$$
\begin{aligned}
& \frac{d}{d t} \int_{\Omega} \omega_{i, j}\left(\omega_{i, j}-\omega_{j, i}\right) d x \\
& \leq\left(2 C^{2}+2 R^{2}\right) \int_{\Omega}\left(\omega_{i, j}-\omega_{j, i}\right)\left(\omega_{i, j}-\omega_{j, i}\right) d x+\frac{1}{2} \int_{\Omega} \hat{\varphi}_{, j} \hat{\varphi}_{, j} d x+\frac{1}{2} \int_{\Omega} \hat{\theta}_{, j} \hat{\theta}_{, j} d x \\
& =\left(4 C^{2}+4 R^{2}\right) \int_{\Omega} \omega_{i, j}\left(\omega_{i, j}-\omega_{j, i}\right) d x+\frac{1}{2} \int_{\Omega} \hat{\varphi}_{, j} \hat{\varphi}_{, j} d x+\frac{1}{2} \int_{\Omega} \hat{\theta}_{, j} \hat{\theta}_{, j} d x .
\end{aligned}
$$

Multiplying both sides of Equation (40) by $2 \hat{\theta}$, and integrating over $\Omega$, we can get:

$$
\begin{aligned}
& \frac{d}{d t} \int_{\Omega} \hat{\theta}^{2} d x=2 \int_{\Omega} \omega_{3} \hat{\theta} d x+2 \int_{\Omega} \hat{\theta} \Delta \hat{\theta} d x-2 \int_{\Omega} \omega_{i} \theta_{,} \hat{\theta} d x-2 \int_{\Omega} u_{i}^{*} \hat{\theta}_{i} \hat{\theta} d x \\
& =2 \int_{\Omega} \omega_{3} \hat{\theta} d x+2 \int_{\Omega} \hat{\theta} \Delta \hat{\theta} d x+2 \int_{\Omega} \omega_{i} \theta \hat{\theta}_{, i} d x .
\end{aligned}
$$

The first term on the right side of Equation (51) can be bounded by:

$$
2 \int_{\Omega} \omega_{3} \hat{\theta} d x \leq \int_{\Omega} \omega_{i} \omega_{i} d x+\int_{\Omega} \hat{\theta}^{2} d x
$$


Using the result (4), and taking $\varepsilon_{0}=1$, we can get:

$$
\int_{\partial \Omega} \hat{\theta}^{2} d S \leq\left(\frac{m_{1}}{m_{0}}+\frac{m_{2}^{2}}{m_{0}^{2}}\right) \int_{\Omega} \hat{\theta}^{2} d x+\int_{\Omega} \hat{\theta}_{,} \hat{\theta}_{i} d x .
$$

We now take the second term on the right side of Equation (51):

$$
\begin{aligned}
2 \int_{\Omega} \hat{\theta} \Delta \hat{\theta} d x & =2 \int_{\partial \Omega} \hat{\theta} \frac{\partial \hat{\theta}}{\partial n} d S-2 \int_{\Omega} \hat{\theta}_{, i} \hat{\theta}_{, i} d x \\
& =2 \hat{k_{1}} \int_{\partial \Omega} \theta \hat{\theta} d S-2 \int_{\Omega} \hat{\theta}_{, i} \hat{\theta}_{, i} d x \\
& \leq \hat{k_{1}^{2}} \int_{\partial \Omega} \theta^{2} d S+\int_{\partial \Omega} \hat{\theta}^{2} d S-2 \int_{\Omega} \hat{\theta}_{, i} \hat{\theta}_{, i} d x \\
& \leq \hat{k_{1}^{2}} \int_{\partial \Omega} \theta^{2} d S+\left(\frac{m_{1}}{m_{0}}+\frac{m_{2}^{2}}{m_{0}^{2}}\right) \int_{\Omega} \hat{\theta}^{2} d x-\int_{\Omega} \hat{\theta}_{, i} \hat{\theta}_{, i} d x .
\end{aligned}
$$

Combining (51), (52), and (54), we can get:

$$
\begin{aligned}
& \frac{d}{d t} \int_{\Omega} \hat{\theta}^{2} d x+\frac{1}{2} \int_{\Omega} \hat{\theta}_{,} \hat{\theta}_{, i} d x \\
& \leq \hat{k}_{1}^{2} \int_{\partial \Omega} \theta^{2} d S+\int_{\Omega} \omega_{i} \omega_{i} d x+m_{5} \int_{\Omega} \hat{\theta}^{2} d x+2 \int_{\Omega} \omega_{i} \omega_{i} \theta^{2} d x \\
& \leq \hat{k}_{1}^{2} \int_{\partial \Omega} \theta^{2} d x+\int_{\Omega} \omega_{i} \omega_{i} d x+m_{5} \int_{\Omega} \hat{\theta}^{2} d x+2\left(\int_{\Omega}\left(\omega_{i} \omega_{i}\right)^{2} d x\right)^{\frac{1}{2}}\left(\int_{\Omega} \theta^{4} d x\right)^{\frac{1}{2}},
\end{aligned}
$$

where $m_{5}=\left(\frac{m_{1}}{m_{0}}+\frac{m_{2}^{2}}{m_{0}^{2}}\right)+1$.

Using the result of (B.17) in [26] and (31), we can get:

$$
\begin{aligned}
& \frac{d}{d t} \int_{\Omega} \hat{\theta}^{2} d x+\frac{1}{2} \int_{\Omega} \hat{\theta}_{i} \hat{\theta}_{i} d x \\
& \leq \hat{k}_{1}^{2} \int_{\partial \Omega} \theta^{2} d S+\int_{\Omega} \omega_{i} \omega_{i} d x+m_{5} \int_{\Omega} \hat{\theta}^{2} d x+2\left(\int_{\Omega}\left(\omega_{i} \omega_{i}\right)^{2} d x\right)^{\frac{1}{2}} n_{4}(t) \\
& \leq \hat{k}_{1}^{2} \int_{\partial \Omega} \theta^{2} d S+\int_{\Omega} \omega_{i} \omega_{i} d x+m_{5} \int_{\Omega} \hat{\theta}^{2} d x+2 M\left\{\frac{5}{4} \int_{\Omega} \omega_{i} \omega_{i} d x+\frac{3}{4} \int_{\Omega} \omega_{i, j} \omega_{i, j} d x\right\} n_{4}(t) \\
& \leq \hat{k}_{1}^{2} \int_{\partial \Omega} \theta^{2} d S+m_{6} \int_{\Omega} \omega_{i} \omega_{i} d x+m_{5} \int_{\Omega} \hat{\theta}^{2} d x+3 M n_{4}(\tau) \int_{\Omega} \omega_{i, j}\left(\omega_{i, j}-\omega_{j, i}\right) d x,
\end{aligned}
$$

where $m_{6}=M n_{4}(\tau)\left[3 k_{0}\left(\frac{m_{1}}{m_{0}}+\frac{2 k_{0} m_{2}^{2}}{m_{0}^{2}}\right)+\frac{5}{2}\right]+1$.

Multiplying both sides of Equation (40) by $2 \hat{\varphi}$, and integrating over $\Omega$ we can get:

$$
\begin{aligned}
\varepsilon_{1} \frac{d}{d t} \int_{\Omega} \hat{\varphi}^{2} d x & =2 \int_{\Omega} \omega_{3} \hat{\varphi} d x+2 \int_{\Omega} \hat{\varphi} \Delta \hat{\varphi} d x-2 L_{e} \int_{\Omega} \omega_{i} \varphi_{, i} \hat{\varphi} d x-2 L_{e} \int_{\Omega} u_{i}^{*} \hat{\psi} \hat{\psi}, i d x \\
& =2 \int_{\Omega} \omega_{3} \hat{\varphi} d x+2 \int_{\Omega} \hat{\varphi} \Delta \hat{\varphi} d x+2 L_{e} \int_{\Omega} \omega_{i} \varphi \hat{\varphi}, i d x .
\end{aligned}
$$

The first term on the right side of Equation (57) can be bounded by:

$$
2 \int_{\Omega} \omega_{3} \hat{\varphi} d x \leq \int_{\Omega} \omega_{i} \omega_{i} d x+\int_{\Omega} \hat{\varphi}^{2} d x .
$$

Using (4), and taking $\varepsilon_{0}=1$, we can get:

$$
\int_{\partial \Omega} \hat{\varphi}^{2} d S \leq\left(\frac{m_{1}}{m_{0}}+\frac{m_{2}^{2}}{m_{0}^{2}}\right) \int_{\Omega} \hat{\varphi}^{2} d x+\int_{\Omega} \hat{\varphi}_{, i} \hat{\varphi}_{, i} d x .
$$


We now take the second term on the right side of Equation (57). We have:

$$
\begin{aligned}
2 \int_{\Omega} \hat{\varphi} \Delta \hat{\varphi} d x & =2 \int_{\partial \Omega} \hat{\varphi} \frac{\partial \hat{\varphi}}{\partial n} d S-2 \int_{\Omega} \hat{\varphi}_{, i} \hat{\varphi}_{, i} d x \\
& =2 \hat{k}_{2} \int_{\partial \Omega} \varphi \hat{\varphi} d S-2 \int_{\Omega} \hat{\varphi}_{, i} \hat{\varphi}_{, i} d x \\
& \leq \hat{k}_{2}^{2} \int_{\partial \Omega} \varphi^{2} d S+\int_{\partial \Omega} \varphi^{2} d S-2 \int_{\Omega} \hat{\varphi}_{, i} \hat{\varphi}_{i} d x \\
& \leq \hat{k}_{2}^{2} \int_{\partial \Omega} \varphi^{2} d S+\left(\frac{m_{1}}{m_{0}}+\frac{m_{2}^{2}}{m_{0}^{2}}\right) \int_{\Omega} \hat{\varphi}^{2} d x-\int_{\Omega} \hat{\varphi}_{, i} \hat{\varphi}_{, i} d x .
\end{aligned}
$$

Combining (57)-(60), we can obtain:

$\varepsilon_{1} \frac{d}{d t} \int_{\Omega} \hat{\varphi}^{2} d x+\frac{1}{2} \int_{\Omega} \hat{\varphi}_{, i} \hat{\varphi}_{, i} d x$

$\leq \hat{k_{2}^{2}} \int_{\partial \Omega} \varphi^{2} d S+\int_{\Omega} \omega_{i} \omega_{i} d x+m_{5} \int_{\Omega} \hat{\varphi}^{2} d x+2 L_{e}^{2} \int_{\Omega} \omega_{i} \omega_{i} \varphi^{2} d x$

$\leq \hat{k}_{2}^{2} \int_{\partial \Omega} \varphi^{2} d S+\int_{\Omega} \omega_{i} \omega_{i} d x+m_{5} \int_{\Omega} \hat{\varphi}^{2} d x+2 L_{e}^{2}\left(\int_{\Omega}\left(\omega_{i} \omega_{i}\right)^{2} d x\right)^{\frac{1}{2}}\left(\int_{\Omega} \varphi^{4} d x\right)^{\frac{1}{2}}$.

We can also get:

$\varepsilon_{1} \frac{d}{d t} \int_{\Omega} \hat{\varphi}^{2} d x+\frac{1}{2} \int_{\Omega} \hat{\varphi}_{, i} \hat{\varphi}_{i} d x$

$\leq \hat{k}_{2}^{2} \int_{\partial \Omega} \varphi^{2} d S+\int_{\Omega} \omega_{i} \omega_{i} d x+m_{5} \int_{\Omega} \hat{\varphi}^{2} d x+2 L_{e}^{2} M\left\{\frac{5}{4} \int_{\Omega} \omega_{i} \omega_{i} d x+\frac{3}{4} \int_{\Omega} \omega_{i, j} \omega_{i, j} d x\right\} n_{5}(t)$

$\leq \hat{k}_{2}^{2} \int_{\partial \Omega} \varphi^{2} d S+m_{7} \int_{\Omega} \omega_{i} \omega_{i} d x+m_{7} \int_{\Omega} \hat{\varphi}^{2} d x+3 M L_{e}^{2} n_{5}(\tau) \int_{\Omega} \omega_{i, j}\left(\omega_{i, j}-\omega_{j, i}\right) d x$,

where $m_{5}=M L_{e}^{2} n_{5}(\tau)\left[3 k_{0}\left(\frac{m_{1}}{m_{0}}+\frac{2 k_{0} m_{2}^{2}}{m_{0}^{2}}\right)+\frac{5}{2}\right]+1$.

Combining (48), (50), (56), (61), and (62), we can get:

$\frac{d}{d t}\left[\int_{\Omega} \omega_{i} \omega_{i} d x+\int_{\Omega} \hat{\theta}^{2} d x+\varepsilon_{1} \int_{\Omega} \hat{\varphi}^{2} d x+\int_{\Omega} \omega_{i, j}\left(\omega_{i, j}-\omega_{j, i}\right) d x\right]$
$\leq\left(m_{6}+m_{7}+2\right) \int_{\Omega} \omega_{i} \omega_{i}+\left(R^{2}+m_{5}\right) \int_{\Omega} \hat{\theta}^{2} d x+\left(C^{2}+m_{5}\right) \int_{\Omega} \hat{\varphi}^{2} d x$
$+\hat{k}_{1}^{2} \int_{\partial \Omega} \theta^{2} d S+\hat{k}_{2}^{2} \int_{\partial \Omega} \varphi^{2} d S+\left(3 M n_{4}(\tau)+3 M L_{e}^{2} n_{5}(\tau)+4 C^{2}+4 R^{2}\right) \int_{\Omega} \omega_{i, j}\left(\omega_{i, j}-\omega_{j, i}\right) d x$.

Let

$$
\begin{gathered}
F_{2}(t)=\int_{\Omega} \omega_{i} \omega_{i} d x+\int_{\Omega} \hat{\theta}^{2} d x+\varepsilon_{1} \int_{\Omega} \hat{\varphi}^{2} d x+\int_{\Omega} \omega_{i, j}\left(\omega_{i, j}-\omega_{j, i}\right) d x, \\
m_{8}=\max \left\{m_{6}+m_{7}+2, R^{2}+m_{5}, \quad \frac{c^{2}+m_{5}}{\varepsilon_{1}}, \quad 3 M n_{4}(\tau)+3 M L_{e}^{2} n_{5}(\tau)+4 C^{2}+4 R^{2} .\right.
\end{gathered}
$$

From (63), it can be seen that:

$$
\frac{d}{d t} F_{2}(t) \leq \hat{k}_{1}^{2} \int_{\partial \Omega} \theta^{2} d S+\hat{k}_{2}^{2} \int_{\partial \Omega} \varphi^{2} d S+m_{8} F_{2}(t) .
$$

Integrating (64), and using (4), (5), (13), and (14), we can get:

$$
F_{2}(t) \leq \hat{k}_{1}^{2} m_{8} e^{m_{8} t} n_{6}(t)+\hat{k}_{2}^{2} m_{8} e^{m_{8} t} n_{7}(t),
$$

with $n_{6}(t)=\int_{0}^{t}\left(\frac{m_{1}}{m_{0}}+\frac{m_{2}^{2}}{m_{0}^{2}}\right) n_{1}(\eta) d \eta+n_{2}(t)$ and $n_{7}(t)=\int_{0}^{t}\left(\frac{m_{1}}{m_{0}}+\frac{m_{2}^{2}}{m_{0}^{2}}\right) n_{1}(\eta) d \eta+n_{3}(t)$. 
Inequality (65) shows that when $\hat{k}_{1}, \hat{k}_{2}$ simultaneously tend to zero, the energy $F_{2}(t)$ also tends to zero as the indicated norm.

\section{Convergence Result for the Lewis Coefficient $L_{e}$}

Let $\left(u_{i}, \theta, \varphi, \pi\right)$ be the solution of (1)-(3) when $L e=\hat{L} e$, and let $\left(u_{i}^{*}, \theta^{*}, \varphi^{*}, \pi^{*}\right)$ be the solution of (1)-(3) when $L e=0$. We assume $\omega_{i}=u_{i}-u_{i}^{*}, \hat{\theta}=\theta-\theta^{*}, \hat{\varphi}=\varphi-\varphi^{*}, \hat{\pi}=$ $\pi-\pi^{*}$, then $\left(\omega_{i}, \hat{\theta}, \hat{\varphi}, \hat{\pi}\right)$ satisfies the following equations:

$$
\begin{array}{r}
\frac{\partial \omega_{i}}{\partial t}=C \hat{\varphi} l_{i}-R \hat{\theta} l_{i}+\hat{\pi}_{, i}, \\
\frac{\partial \omega_{i}}{\partial x_{i}}=0, \\
\frac{\partial \hat{\theta}}{\partial t}+\omega_{i} \theta_{, i}+u_{i}^{*} \hat{\theta}_{, i}=\omega_{3}+\Delta \hat{\theta}, \\
\varepsilon_{1} \frac{\partial \hat{\varphi}}{\partial t}+\hat{L}_{e} u_{i} \varphi_{, i}=\omega_{3}+\Delta \hat{\varphi} .
\end{array}
$$

The boundary conditions are:

$$
\omega_{i} n_{i}=0, \quad \frac{\partial \hat{\theta}}{\partial n}=k_{1} \hat{\theta}, \quad \frac{\partial \hat{\varphi}}{\partial n}=k_{2} \hat{\varphi}, \quad(x, t) \in \partial \Omega \times[0, \tau] .
$$

The initial conditions are:

$$
\omega_{i}(x, 0)=0, \hat{\varphi}(x, 0)=0, \hat{\theta}(x, 0)=0, x \in \Omega .
$$

Theorem 2. Let $\left(u_{i}, \theta, \varphi, \pi\right)$ be the solution of (1)-(3) when Le $=\hat{L e},\left(u_{i}^{*}, \theta^{*}, \varphi^{*}, \pi^{*}\right)$ be the solution of (1)-(3) when Le $=0$. We assume $\omega_{i}=u_{i}-u_{i}^{*}, \hat{\theta}=\theta-\theta^{*}, \hat{\varphi}=\varphi-\varphi^{*}, \hat{\pi}=\pi-\pi^{*}$, then $\left(\omega_{i}, \hat{\theta}, \hat{\varphi}, \hat{\pi}\right)$ satisfies the following estimates:

$$
\begin{aligned}
& \int_{\Omega} \omega_{i} \omega_{i} d x+\int_{\Omega} \hat{\theta}^{2} d x+\varepsilon_{1} \int_{\Omega} \hat{\varphi}^{2} d x+\int_{\Omega} \omega_{i, j}\left(\omega_{i, j}-\omega_{j, i}\right) d x \\
& \leq 2 \hat{L}_{e}^{2} m_{11} e^{m_{11} t} \int_{0}^{t} m_{1}(\eta) n_{5}(\eta) e^{-m_{11} \eta} d \eta
\end{aligned}
$$

where $m_{11}$ is a constant greater than zero.

Proof. Multiplying both sides of Equation (66) by $2 \omega_{i}$, and integrating over $\Omega \times[0, t]$, we can obtain:

$$
\begin{aligned}
\frac{d}{d t} \int_{\Omega} \omega_{i} \omega_{i} d x & =2 C \int_{\Omega} \hat{\varphi} l_{i} \omega_{i} d x-2 R \int_{\Omega} \hat{\theta} l_{i} \omega_{i} d x+2 \int_{\Omega} \hat{\pi}_{, i} \omega_{i} d x \\
& \leq 2 \int_{\Omega} \omega_{i} \omega_{i} d x+C^{2} \int_{\Omega} \hat{\varphi}^{2} d x+R^{2} \int_{\Omega} \hat{\theta}^{2} d x
\end{aligned}
$$

Multiplying both sides of Equation (66) by $2 \hat{\theta}$, and integrating over $\Omega$, we can get,

$$
\begin{aligned}
& \frac{d}{d t} \int_{\Omega} \hat{\theta}^{2} d x=2 \int_{\Omega} \omega_{3} \hat{\theta} d x+2 \int_{\Omega} \hat{\theta} \Delta \hat{\theta} d x-2 \int_{\Omega} \omega_{i} \theta_{i} \hat{\theta} d x-2 \int_{\Omega} u_{i}^{*} \hat{\theta}_{,} \hat{\theta} d x \\
& =2 \int_{\Omega} \omega_{3} \hat{\theta} d x+2 \int_{\Omega} \hat{\theta} \Delta \hat{\theta} d x+2 \int_{\Omega} \omega_{i} \theta \hat{\theta}_{, i} d x .
\end{aligned}
$$

The first term on the right side of Equation (71) can be obtained from Hölder's inequality:

$$
2 \int_{\Omega} \omega_{3} \hat{\theta} d x \leq \int_{\Omega} \omega_{i} \omega_{i} d x+\int_{\Omega} \hat{\theta}^{2} d x
$$


Using a method similar to (4), and taking $\varepsilon_{0}=2 k_{1}$, we can get:

$$
\int_{\partial \Omega} \hat{\theta}^{2} d S \leq\left(\frac{m_{1}}{m_{0}}+2 k_{1} \frac{m_{2}^{2}}{m_{0}^{2}}\right) \int_{\Omega} \hat{\theta}^{2} d x+\int_{\Omega} \hat{\theta}_{, i} \hat{\theta}_{i} d x .
$$

We now deal with the second term on the right side of Equation (71). We have:

$$
\begin{aligned}
2 \int_{\Omega} \hat{\theta} \Delta \hat{\theta} d x & =2 \int_{\partial \Omega} \hat{\theta} \frac{\partial \hat{\theta}}{\partial n} d S-2 \int_{\Omega} \hat{\theta}_{, i} \hat{\theta}_{, i} d x \\
& =2 k_{1} \int_{\partial \Omega} \hat{\theta}^{2} d S-2 \int_{\Omega} \hat{\theta}_{, i} \hat{\theta}_{, i} d x \\
& \leq \hat{k}_{1}^{2} \int_{\partial \Omega} \hat{\theta}^{2} d S-2 \int_{\Omega} \hat{\theta}_{, i} \hat{\theta}_{, i} d x \\
& \leq 2 k_{1}\left(\frac{m_{1}}{m_{0}}+2 k_{1} \frac{m_{2}^{2}}{m_{0}^{2}}\right) \int_{\Omega} \hat{\theta}^{2} d x-\int_{\Omega} \hat{\theta}_{, i} \hat{\theta}_{i} d x
\end{aligned}
$$

Combining (71)-(74), we can get:

$$
\begin{aligned}
& \frac{d}{d t} \int_{\Omega} \hat{\theta}^{2} d x+\frac{1}{2} \int_{\Omega} \hat{\theta}_{, i} \hat{\theta}_{, i} d x \\
& \leq \int_{\Omega} \omega_{i} \omega_{i} d x+m_{9} \int_{\Omega} \hat{\theta}^{2} d x+2 \int_{\Omega} \omega_{i} \omega_{i} \theta^{2} d x \\
& \leq \int_{\Omega} \omega_{i} \omega_{i} d x+m_{9} \int_{\Omega} \hat{\theta}^{2} d x+2\left(\int_{\Omega}\left(\omega_{i} \omega_{i}\right)^{2} d x\right)^{\frac{1}{2}}\left(\int_{\Omega} \theta^{4} d x\right)^{\frac{1}{2}},
\end{aligned}
$$

where $m_{9}=2 k_{1}\left(\frac{m_{1}}{m_{0}}+2 k_{1} \frac{m_{2}^{2}}{m_{0}^{2}}\right)+1$.

Using the result of (B.17) in [26] again, we can also get:

$$
\begin{aligned}
& \frac{d}{d t} \int_{\Omega} \hat{\theta}^{2} d x+\frac{1}{2} \int_{\Omega} \hat{\theta}_{, i} \hat{\theta}_{, i} d x \\
& \leq \int_{\Omega} \omega_{i} \omega_{i} d x+m_{9} \int_{\Omega} \hat{\theta}^{2} d x+2\left(\int_{\Omega}\left(\omega_{i} \omega_{i}\right)^{2} d x\right)^{\frac{1}{2}} n_{4}(t) \\
& \leq \int_{\Omega} \omega_{i} \omega_{i} d x+m_{9} \int_{\Omega} \hat{\theta}^{2} d x+2 M\left\{\frac{5}{4} \int_{\Omega} \omega_{i} \omega_{i} d x+\frac{3}{4} \int_{\Omega} \omega_{i, j} \omega_{i, j} d x\right\} n_{4}(t) \\
& \leq m_{6} \int_{\Omega} \omega_{i} \omega_{i} d x+m_{9} \int_{\Omega} \hat{\theta}^{2} d x+3 M n_{4}(\tau) \int_{\Omega} \omega_{i, j}\left(\omega_{i, j}-\omega_{j, i}\right) d x .
\end{aligned}
$$

Multiplying both sides of Equation (66) ${ }_{3}$ by $2 \hat{\varphi}$, and integrating over $\Omega \times[0, t]$, we can get,

$$
\begin{aligned}
\varepsilon_{1} \frac{d}{d t} \int_{\Omega} \hat{\varphi}^{2} d x & =2 \int_{\Omega} \omega_{3} \hat{\varphi} d x+2 \int_{\Omega} \hat{\varphi} \Delta \hat{\varphi} d x-2 \hat{L_{e}} \int_{\Omega} u_{i} \varphi_{, i} \hat{\varphi} d x \\
& =2 \int_{\Omega} \omega_{3} \hat{\varphi} d x+2 \int_{\Omega} \hat{\varphi} \Delta \hat{\varphi} d x+2 \hat{L}_{e} \int_{\Omega} u_{i} \varphi \hat{\varphi}{ }_{, i} d x
\end{aligned}
$$

The first term on the right side of Equation (77) can be bounded by:

$$
2 \int_{\Omega} \omega_{3} \hat{\varphi} d x \leq \int_{\Omega} \omega_{i} \omega_{i} d x+\int_{\Omega} \hat{\varphi}^{2} d x .
$$

Using the result (4), and taking $\varepsilon_{0}=2 k_{2}$, we can get:

$$
\int_{\partial \Omega} \hat{\varphi}^{2} d S \leq\left(\frac{m_{1}}{m_{0}}+2 k_{2} \frac{m_{2}^{2}}{m_{0}^{2}}\right) \int_{\Omega} \hat{\varphi}^{2} d x+\int_{\Omega} \hat{\varphi}_{, i} \hat{\varphi}_{, i} d x .
$$


The second term on the right side of (77) can be bounded as follows:

$$
\begin{aligned}
2 \int_{\Omega} \hat{\varphi} \Delta \hat{\varphi} d x & =2 \int_{\partial \Omega} \hat{\varphi} \frac{\partial \hat{\varphi}}{\partial n} d S-2 \int_{\Omega} \hat{\varphi}_{, i} \hat{\varphi}_{, i} d x \\
& =2 k_{2} \int_{\partial \Omega} \hat{\varphi}^{2} d S-2 \int_{\Omega} \hat{\varphi}_{, i} \hat{\varphi}_{, i} d x \\
& \leq 2 k_{2}\left(\frac{m_{1}}{m_{0}}+2 k_{2} \frac{m_{2}^{2}}{m_{0}^{2}}\right) \int_{\Omega} \hat{\varphi}^{2} d x-\int_{\Omega} \hat{\varphi}_{, i} \hat{\varphi}_{, i} d x .
\end{aligned}
$$

Combining (77), (78), and (80), we can obtain:

$$
\begin{aligned}
& \varepsilon_{1} \frac{d}{d t} \int_{\Omega} \hat{\varphi}^{2} d x+\frac{1}{2} \int_{\Omega} \hat{\varphi}_{, i} \hat{\varphi}_{, i} d x \\
& \leq \int_{\Omega} \omega_{i} \omega_{i} d x+m_{10} \int_{\Omega} \hat{\varphi}^{2} d x+2{\hat{L_{e}}}^{2} \int_{\Omega} u_{i} u_{i} \varphi^{2} d x \\
& \leq \int_{\Omega} \omega_{i} \omega_{i} d x+m_{10} \int_{\Omega} \hat{\varphi}^{2} d x+2{\hat{L_{e}}}^{2}\left(\int_{\Omega}\left(u_{i} u_{i}\right)^{2} d x\right)^{\frac{1}{2}}\left(\int_{\Omega} \varphi^{4} d x\right)^{\frac{1}{2}},
\end{aligned}
$$

where $m_{10}=2 k_{2}\left(\frac{m_{1}}{m_{0}}+2 k_{2} \frac{m_{2}^{2}}{m_{0}^{2}}\right)+1$.

Using the results (23) and (32), we can obtain:

$$
\begin{aligned}
& \varepsilon_{1} \frac{d}{d t} \int_{\Omega} \hat{\varphi}^{2} d x+\frac{1}{2} \int_{\Omega} \hat{\varphi}_{, i} \hat{\varphi}_{, i} d x \\
& \leq \int_{\Omega} \omega_{i} \omega_{i} d x+m_{10} \int_{\Omega} \hat{\varphi}^{2} d x+2 \hat{L}_{e}^{2} m_{1}(t) n_{5}(t) .
\end{aligned}
$$

A combination of (70), (76), (82), and (50) gives:

$$
\begin{aligned}
& \frac{d}{d t}\left[\int_{\Omega} \omega_{i} \omega_{i} d x+\int_{\Omega} \hat{\theta}^{2} d x+\varepsilon_{1} \int_{\Omega} \hat{\varphi}^{2} d x+\int_{\Omega} \omega_{i, j}\left(\omega_{i, j}-\omega_{j, i}\right) d x\right] \\
& \leq\left(m_{6}+3\right) \int_{\Omega} \omega_{i} \omega_{i}+\left(R^{2}+m_{9}\right) \int_{\Omega} \hat{\theta}^{2} d x+\left(C^{2}+m_{10}\right) \int_{\Omega} \hat{\varphi}^{2} d x \\
& +\left(3 M n_{4}(\tau)+4 C^{2}+4 R^{2}\right) \int_{\Omega} \omega_{i, j}\left(\omega_{i, j}-\omega_{j, i}\right) d x+2 \hat{L}_{e}{ }^{2} m_{1}(t) n_{5}(t) .
\end{aligned}
$$

Let,

$$
\begin{gathered}
F_{3}(t)=\int_{\Omega} \omega_{i} \omega_{i} d x+\int_{\Omega} \hat{\theta}^{2} d x+\varepsilon_{1} \int_{\Omega} \hat{\varphi}^{2} d x+\int_{\Omega} \omega_{i, j}\left(\omega_{i, j}-\omega_{j, i}\right) d x, \\
m_{11}=\max \left\{m_{6}+3, \quad R^{2}+m_{9}, \frac{C^{2}+m_{10}}{\varepsilon_{1}}, \quad 3 M n_{4}(\tau)+4 C^{2}+4 R^{2}\right\} .
\end{gathered}
$$

From (83), it can be seen that:

$$
\frac{d}{d t} F_{3}(t) \leq 2 \hat{L}_{e}^{2} m_{1}(t) n_{5}(t)+m_{11} F_{2}(t)
$$

by an integration of (84) leads to:

$$
F_{3}(t) \leq 2 \hat{L}_{e}^{2} m_{11} e^{m_{11} t} \int_{0}^{t} m_{1}(\eta) n_{5}(\eta) e^{-m_{11} \eta} d \eta .
$$

Inequality (85) shows that when $\hat{L}_{e}$ tends to zero, the energy $F_{3}(t)$ also tends to zero.

\section{Conclusions}

In this paper, we studied the convergence results for the double-diffusion perturbation equations in a bounded domain. The convergence result of solutions were gained for 
the reaction boundary coefficients $k_{1}, k_{2}$ and the Lewis coefficient $L_{e}$. Using the method in this paper, similar results for other coefficients could also be gained. Our method is useful for studying the structural stability of bounded regions. However, for unbounded regions, because the regions become more complex, and the inequalities that can be used in bounded regions cannot be used in unbounded regions, essential difficulties will arise. Methods of dealing with stress terms will be the biggest difficulty in unbounded areas. It is an open problem now that we could solve by constructing special functions in relevant future research. In this paper, we only give a theoretical proof and a numerical simulation will be given in another paper.

Author Contributions: Writing — original draft, S.L. and J.S. All authors have read and agreed to the published version of the manuscript.

Funding: This research was funded by key projects of universities in the Guangdong Province (Natural Science) (2019KZDXM042), "Thirteenth Five-Year Plan" 2020 research project approval (2020JKDY040), and the Research Foundations of Guangzhou Huashang College (2021HSKT01, 2020HSDS16).

Institutional Review Board Statement: Not applicable.

Informed Consent Statement: Not applicable.

Data Availability Statement: Not applicable.

Acknowledgments: The authors would like to deeply thank all the reviewers for their insightful and constructive comments.

Conflicts of Interest: The authors declare no conflict of interest.

\section{References}

1. Ames, K.A.; Straughan, B. Non-Standard and Improperly Posed Problems; Mathematics in Science and Engineering series; Academic Press: San Diego, CA, USA, 1997; Volume 194.

2. Hirsch, M.W.; Smale, S. Differential Equations, Dynamical Systems, and Linear Algebra; Academic Press: New York, NY, USA, 1974.

3. Straughan, B. Heated and salted below porous convection with generalized temperature and solute boundary conditions. Transp. Porous Media 2020, 131, 617-631. [CrossRef]

4. Straughan, B. Stability and Wave Motion in Porous Media; Springer Science \& Business Media: Berlin/Heidelberg, Germany, 2008; Volume 165.

5. Straughan, B. Anisotropic inertia effect in microfluidic porous thermosolutal convection. Microfluid. Nanofluidics 2014, 16, 361-368. [CrossRef]

6. $\quad$ Nield, D.A.; Bejan, A. Convection in Porous Media; Springer: New York, NY, USA, 1992.

7. Straughan, B. Continuous Dependence on the Heat Source in Resonant Porous Penetrative Convection. Stud. Appl. Math. 2011, 127, 302-314. [CrossRef]

8. Payne, L.E.; Song, J.C. Spatial decay estimates for the Brinkman and Dracy flows in a semi-infinite cylinder. Contin. Mech. Thermodyn. 1997, 9, 175-190. [CrossRef]

9. Payne, L.E.; Song, J.C. Spatial decay bounds for double diffusive convection in Brinkman flow. J. Differ. Equ. 2008, 244, 413-430. [CrossRef]

10. Ames, K.A.; Payne, L.E.; Song, J.C. Spatial decay in the pipe flow of a viscous fluid interfacing a porous medium. Math. Models Meth. Appl. Sci. 2001, 11, 1547-1562. [CrossRef]

11. Payne, L.E.; Straughan, B. Stability in the initial-time geometry problem for the Brinkman and Darcy equations of flow in porous media. J. Math. Pures Appl. 1996, 75, 255-271.

12. Payne, L.E.; Straughan, B. Structural stability for the Darcy equations of flow in porous media. Proc. R. Soc. Lond. A 1998, 454 , 1691-1698. [CrossRef]

13. Payne, L.E.; Straughan, B. Convergenc and continuous dependence for the Brinkman-Forchheimer equations. Stud. Appl. Math. 1999, 102, 419-439. [CrossRef]

14. Scott, N.L. Continuous dependence on boundary reaction terms in a porous medium of Darcy type. J. Math. Anal. Appl. 2013, 399, 667-675. [CrossRef]

15. Scott, N.L.; Straughan, B. Continuous dependence on the reaction terms in porous convection with surface reactions. Quart. Appl. Math. 2013, 71, 501-508. [CrossRef]

16. Straughan, B. The Energy Method, Stability and Nonlinear Convection; Springer Science \& Business Media: Berlin/Heidelberg, Germany, 2004; Volume 91. 
17. Ames, K.A.; Payne, L.E. On stabilizing against modelling errors in a penetrative convection problem for a porous medium. Model. Methods Appl. Sci. 1990, 4, 733-740. [CrossRef]

18. Celebi, A.O.; Kalantarov, V.K.; Ugurlu, D. Continuous dependence for the convective Brinkman-Forchheimer equations. Appl. Anal. 2005, 84, 877-888. [CrossRef]

19. Celebi, A.O.; Kalantarov, V.K.; Ugurlu, D. On continuous dependence on coefficients of the Brinkman-Forchheimer equations. Appl. Math. Lett. 2006, 19, 801-807. [CrossRef]

20. Franchi, F.; Straughan, B. Continuous dependence and decay for the Forchheimer equations. Proc. R. Soc. Lond. A 2003, 459, 3195-3202. [CrossRef]

21. Harfash, A.J. Structural Stability for Two Convection Models in a Reacting Fluid with Magnetic Field Effect. Ann. Henri Poincare 2014, 15, 2441-2465. [CrossRef]

22. Kaloni, P.N.; Guo, J. Steady nonlinear double-diffusive convection in a porous medium based upon the Brinkman-Forchheimer Model. J. Math. Anal. Appl. 1996, 204, 138-155. [CrossRef]

23. Li, Y.; Lin, C. Continuous dependence for the nonhomogeneous Brinkman-Forchheimer equations in a semi-infinite pipe. Appl. Math. Comput. 2014, 244, 201-208. [CrossRef]

24. Lin, C.; Payne, L.E. Structural stability for a Brinkman fluid. Math. Meth. Appl. Sci. 2007, 30, 567-578. [CrossRef]

25. Lin, C.; Payne, L.E. Structural stability for the Brinkman equations of flow in double diffusive convection. J. Math. Anal. Appl. 2007, 325, 1479-1490. [CrossRef]

26. Lin, C.; Payne, L.E. Continuous dependence on the Soret coefficient for double diffusive convection in Darcy flow. J. Math. Anal. Appl. 2008, 342, 311-325. [CrossRef]

27. Payne, L.E.; Song, J.C.; Straughan, B. Continuous dependence and convergence results for Brinkman and Forchheimer models with variable viscosity. Proc. R. Soc. Lond. A 1999, 45S, 2173-2190. [CrossRef]

28. Straughan, B.; Hutter, K. A priori bounds and structural stability for double diffusive convection incorporating the Soret effect. Proc. R. Soc. Lond. A 1999, 455, 767-777. [CrossRef]

29. Gentile, M.; Straughan, B. Structural stability in resonant penetrative convection in a Forchheimer porous material. Nonlinear Anal. Real. World Appl. 2013, 14, 397-401. [CrossRef]

30. Horgan, L.; Ibragimov, A. Structural stability of generalized Forchheimer equations for Compressible fuids in porous media. Nonlinearity 2011, 24, 1-41.

31. Li, Y.F.; Xiao, S.Z.; Zeng, P. The applications of some basic mathematical inequalities on the convergence of the primitive equations of moist atmosphere. J. Math. Inequalities 2021, 15, 293-304. [CrossRef]

32. Liu, Y. Continuous dependence for a thermal convection model with temperaturedependent solubitity. Appl. Math. Comput. 2017, $308,18-30$.

33. Payne, L.E.; Straughan, B. Analysis of the boundary condition at the interface between a viscous fluid and a porous medium and related modelling questions. J. Math. Pures Appl. 1998, 77, 317-354. [CrossRef]

34. Li, Y.F.; Zhang, S.H.; Lin, C.L. Structural stability for the Boussinesq equations interfacing with Darcy equations in a bounded domain. Bound. Value Probl. 2021, 27, 1-19. [CrossRef]

35. Liu, Y.; Xiao, S.; Lin, Y.W. Continuous dependence for the Brinkman-Forchheimer fluid interfacing with a Darcy fluid in a bounded domain. Math. Comput. Simul. 2018, 150, 66-82. [CrossRef]

36. Shi, J.C.; Liu, Y. Structural stability for the Forchheimer equations interfacing with a Darcy fluid in a bounded region in $R^{3}$. Bound. Value Probl. 2021, 46, 1-22.

37. Ciarletta, M.; Straughan, B.; Tibullo, V. Structural stability for a thermal convection model with temperature-dependent solubility. Nonlinear Anal. Real World Appl. 2015, 22, 34-43. [CrossRef]

38. Chen, W.; Palmieri, A. Nonexistence of global solutions for the semilinear Moore-Gibson-Thompson equation in the conservative case. Discret. Contin. Dyn. Syst. 2020, 40, 5513-5540. [CrossRef]

39. Chen, W.; Ikehata, R. The Cauchy problem for the Moore-Gibson-Thompson equation in the dissipative case. J. Differ. Equ. 2021, 292, 176-219. [CrossRef]

40. Palmieri, A.; Takamura, H. Blow-up for a weakly coupled system of semilinear damped wave equations in the scattering case with power nonlinearities. Nonlinear Anal. 2019, 187, 467-492. [CrossRef]

41. Palmieri, A.; Reissig, M. Semi-linear wave models with power non-linearity and scale-invariant time-dependent mass and dissipation, II. Math. Nachr. 2018, 291, 1859-1892. [CrossRef]

42. Liu, Y.; Chen, W. Asymptotic profiles of solutions for regularity-loss-type generalized thermoelastic plate equations and their applications. Z. Angew. Math. Phys. 2020, 71, 1-14. [CrossRef]

43. Liu, Y.; Li, Y.; Shi, J. Estimates for the linear viscoelastic damped wave equation on the Heisenberg group. J. Differ. Equ. 2021, 285, 663-685. [CrossRef]

44. Weatherburn, C.E. Differential Geometry of Three Dimensions; Cambrige University Press: Cambrige, UK, 1980.

45. Chen, W. Cauchy problem for thermoelastic plate equations with different damping mechanisms. Commun. Math. Sci. 2020, 18, 429-457. [CrossRef]

46. Chen, W. Decay properties and asymptotic profiles for elastic waves with Kelvin-Voigt damping in 2D. Asymptot. Anal. 2020, 117, 113-140. [CrossRef] 
47. Chen, W.; Palmieri, A. Weakly coupled system of semilinear wave equations with distinct scale-invariant terms in the linear part. Z. Angew. Math. Phys. 2019, 70, 67. [CrossRef]

48. Chen, W.; Palmier, A. A blow-up result for the semilinear Moore-Gibson-Thompson equation with nonlinearity of derivative type in the conservative case. arXiv 2019, arXiv:1909.09348. [CrossRef] 